Kansas State University Libraries

New Prairie Press

\title{
ESTIMATING RHEOLOGICAL PROPERTIES OF YOGURT USING DIFFERENT VERSIONS OF THE FREUNDLICH MODEL AND DESIGN MATRICES
}

M. Zhou
A. M. Parkhurst
H. K. Voss
C. L. Weller

See next page for additional authors

Follow this and additional works at: https://newprairiepress.org/agstatconference

Part of the Agriculture Commons, and the Applied Statistics Commons

\section{(c) (1) $\Theta($}

This work is licensed under a Creative Commons Attribution-Noncommercial-No Derivative Works 4.0 License.

\section{Recommended Citation}

Zhou, M.; Parkhurst, A. M.; Voss, H. K.; and Weller, C. L. (2004). "ESTIMATING RHEOLOGICAL PROPERTIES OF YOGURT USING DIFFERENT VERSIONS OF THE FREUNDLICH MODEL AND DESIGN MATRICES," Conference on Applied Statistics in Agriculture. https://doi.org/10.4148/2475-7772.1160 inclusion in Conference on Applied Statistics in Agriculture by an authorized administrator of New Prairie Press. For more information, please contact cads@k-state.edu. 
Author Information

M. Zhou, A. M. Parkhurst, H. K. Voss, and C. L. Weller

This is available at New Prairie Press: https://newprairiepress.org/agstatconference/2004/proceedings/14 


\title{
ESTIMATING RHEOLOGICAL PROPERTIES OF YOGURT USING DIFFERENT VERSIONS OF THE FREUNDLICH MODEL AND DESIGN MATRICES
}

\author{
M. Zhou ${ }^{1}$, A. M. Parkhurst ${ }^{1}$, H. K. Voss ${ }^{2}$, and C. L. Weller ${ }^{3}$ \\ 1. Department of Statistics, University of Nebraska at Lincoln \\ 2. Department of Food Science and Technology, University of Nebraska at Lincoln \\ 3. Department of Biological Systems Engineering, University of Nebraska at Lincoln
}

\begin{abstract}
The rheological properties described by the consistency coefficient and flow behavior index can be estimated from the relationship between shear stress and shear strain rate following a Freundlich model. An additional rheological property of concern to food scientists studying yogurt is yield stress. They extend the Freundlich model to include a three-parameter model called the Herschel-Bulkley model. In addition, the Herschel-Bulkley model is often linearized by taking logarithms of both sides. An additional complication is the viscometer limits the range of shear strain rates. The objectives of this study are to compare parameter estimates from the three models and to investigate the effects of different designs on the models.
\end{abstract}

\section{INTRODUCTION}

Rheology is the science which deals with the deformation and flow of matter. It is the study of the manner in which materials respond to applied stress or strain. All materials have rheological properties.

Rheological properties of yogurt can be described by yield stress, consistency coefficient, and flow behavior index. Yield stress is the minimum shear stress which is required to initiate the flow of yogurt. The consistency coefficient is a measure of yogurt's resistance to flow. The flow behavior index actually characterizes the rheological nature of a material. Different materials have different flow behavior indices. For example: the index of water is 1.00 ; the index of $40 \%$ raw corn starch solution is greater than 1.00; and, the index of yogurt in this study is between 0 and 1.00. A material with a flow behavior index of 1.00 is a Newtonian material. By definition, the ratio of shear stress (applied force) to shear rate (flow or deformation) for a Newtonian material remains constant when the material undergoes deformation (flow). Yogurt in the study was non-Newtonian in nature because its flow behavior index does not equal 1.00 .

Since yogurt in the study is a non-Newtonian fluid, its rheological properties can be estimated from the relationship between shear stress and shear strain rate by the Herschel-Bulkley model (Steffe, 1996), which is an extension of Freundlich model to a three-parameter model in the field of statistics:

$$
Y=\delta+\beta \cdot X^{\tau}+\varepsilon \quad \varepsilon \sim N\left(0, I \sigma^{2}\right)
$$

Where $\mathrm{Y}$ is shear stress $(\mathrm{Pa}), \mathrm{X}$ is shear strain rate $(1 / \mathrm{sec}), \delta$ is yield stress $(\mathrm{Pa}), \beta$ is consistency coefficient $\left(\mathrm{Pa} \cdot \mathrm{s}^{\mathrm{n}}\right)$, and $\tau$ is flow behavior index. 
Some properties of the 3-parameter Freundlich model are: 1) the rate of change of Y is not constant, rather it is proportional to a power of the current shear strain rate, $\mathrm{X}$,

$$
\frac{\partial Y}{\partial X}=\beta \cdot \tau \cdot X^{\tau-1}
$$

2) $\delta$ is the value of $Y$ at $X=0$; 3) $\beta \cdot \tau$ is the proportionality constant; and 4) $\tau$ is the power constant.

The patterns governed by the parameters can be seen in Figures 1 and 2. Figure 1 focuses on the flow behavior index, $\tau$, and shows the relationship between $\mathrm{X}$, and $\mathrm{Y}$, when the consistency coefficient, $\beta$ is held constant. As $\tau$ increases to 1 , the initial curvature becomes less prominent finally dissolving to a straight line when $\tau$ equals 1 . Figure 2 focuses on $\beta$, the consistency coefficient and the pattern between $\mathrm{X}$ and $\mathrm{Y}$ for a constant value of $\tau$. After the initial curvature, the rate of increase steepens sharply in a linear fashion for higher $\beta(>1)$.

Researchers in food science estimate the rheological parameters of yogurt using the following procedure. First, the data is plotted, the curve is extended to the Y-axis, and the $\mathrm{Y}$-intercept is estimated visually as yield stress, $\delta$. Then, the Herschel-Bulkley model is reduced to a 2-parameter model and is linearized by taking logarithms of both sides:

$$
\ln (Y-\delta)=\ln (\beta)+\tau \cdot \ln (X)+\varepsilon^{*}
$$

Where the $\varepsilon^{*}$ are assumed identical and independent normally distributed with mean 0 and variance $\sigma^{*^{2}}$. Finally, a linear regression of $\ln (\mathrm{Y}-\delta)$ as a function of $\ln (\mathrm{X})$ is fitted to estimate $\beta$ and $\tau$. This procedure of estimating rheological parameters of yogurt has several problems. First, yield stress, $\delta$, is visually estimated. This choice of $\delta$ greatly influences the estimates $\beta$ and $\tau$. Finally, bias is introduced when linearizing a nonlinear model if the errors are additive.

The objectives of this study are: to illustrate problems with the conventional method of estimation; to find estimates for the 3-parameter Freundlich model and to study their validity; to compare the 3-parameter Freundlich model with its expected value parameterization; and, to investigate different choices of shear strain rates, $\mathrm{X}$.

\section{Materials and Methods}

\section{2.a Data}

H. K. Voss (2002) studied the effect of Fructooligosaccharides (FOS) on the rheological properties of yogurt. Yogurt was made on two separate occasions (Tuesday and Friday) using the same method. It was then divided into five portions, and either $0,1,2,3$, or $4 \%(\mathrm{wt} / \mathrm{vol})$ FOS was added. An automatic filler was then used to dispense the yogurt mixtures into individual containers. The containers were incubated at 43 to $45^{\circ} \mathrm{C}$ until the $\mathrm{pH}$ of selected samples reached $4.4 \pm 0.03$. Yogurt containers were then transferred to a cooler $\left(2\right.$ to $\left.4^{\circ} \mathrm{C}\right)$ and held for the length of the study. For the rheological analyses, yogurt samples were randomly taken from the storage cooler $18( \pm 6) \mathrm{h}$ after incubation ended. These samples were designated as the time zero samples. Yogurt samples were subsequently tested at weekly intervals for four weeks. A Brookfield Viscometer (model RVT DV-I, Middleboro, MA) was 
used to obtain values of shear stress and shear strain rate for each yogurt sample. Then the consistency coefficient, $\beta$, flow behavior index, $\tau$, and yield shear stress, $\delta$, were determined for each yogurt sample using Eq.1. A \#2 LV-type spindle was used and measurements were taken every $10 \mathrm{~s}$ at 6 separate rpm settings. The entire study was replicated, and for each test of the rheological properties, duplicate samples were made. All yogurt samples in this study were analyzed but only one yogurt blocktreatment-sample over time is used as an illustration in this paper (Table 1).

\section{2.b Simulation Studies}

Simulation studies were conducted to illustrate the problems with the conventional estimation procedure and to investigate the coverage of the joint confidence regions and simultaneous confidence intervals. First, a Freundlich model was fit to the data (Table 1) using PROC NLIN in SAS (1999). The nonlinear least squares estimates, SE's, and CI's were obtained for all parameters, as was the residual mean squares, MSE. These estimates were used to generate 1000 data sets of size $n=6$. The values of $X(0.21,0.54,1.09,2.19,4.37,10.93)$ were the same for each simulated data set. The error distribution was chosen to be additive and normally distributed with mean zero and variance equal to MSE. The SAS function, RANNOR with a given seed generated the standardized random errors to be multiplied by the MSE.

\section{2.b.i Simulation used to Show the Problems with the Conventional Procedure}

The 3-parameter Freundlich model was fitted. Then for each of the 1000 data sets, the rheological parameters of yogurt were estimated by the conventional procedure. But, instead of visual estimation, $\delta$ was estimated by fitting a linear regression of $\mathrm{Y}$ as a function of $X$ using the first three observations (PROC REG, SAS 1999). The intercept in the linear model was used as an estimate of $\delta$. Finally, a linear regression of $\ln (\mathrm{Y}-\delta$ ) as a function of $\ln (\mathrm{X})$ was fitted to estimate $\beta$ and $\tau$ (PROC REG, SAS 1999).

\section{2.b.ii Simulations used to Calculate the True Coverage Level of Joint Confidence Regions (JCR) and Simultaneous Confidence Intervals (SCI)}

The parameter estimates for both the 3-parameter Freundlich model and its expected value parameterization were obtained for each of the 1000 simulated data sets. PROC NLIN (SAS 1999) was used to fit the nonlinear model. Then Eq.4 and Eq.5 were checked to see whether or not the true values of the parameters were inside the $95 \%$ simultaneous confidence intervals and Eq.6 was checked to see whether or not the true values of the parameters were inside the 95\% JCR. The difference between Eq. 4 and Eq. 5 is that Eq. 4 constructs the simultaneous confidence intervals using univariate confidence intervals. For $\mathrm{p}$ confidence intervals considered simultaneously, the confidence coefficient $(1-\alpha)^{\mathrm{p}}$ is much lower. Bonferroni method (Eq.5) used here gives a lower bound for the confidence coefficient.

$$
\begin{array}{lc}
-t_{\alpha / 2, \nu} S E(\hat{\theta}) \leq \theta-\hat{\theta} \leq t_{\alpha / 2, \nu} S E(\hat{\theta}) & \text { Eq.4 } \\
-t_{\alpha /(2 p), \nu} S E(\hat{\theta}) \leq \theta-\hat{\theta} \leq t_{\alpha /(2 p), v} S E(\hat{\theta}) & \text { Eq.5 } \\
(\theta-\hat{\theta})^{\prime} V^{\prime} V(\theta-\hat{\theta}) \leq p F_{\alpha, p, v} s^{2} & \text { Eq.6 }
\end{array}
$$


Where $\theta$ are true values of parameters, $\hat{\theta}$ are simulated estimates of parameters, $\operatorname{SE}(\hat{\theta})$ are standard errors of estimates, $\mathrm{V}$ is the $\mathrm{p} \mathrm{x} \mathrm{n}$ matrix of the first derivatives evaluated at the simulated estimates, $p$ is the number of parameters, $\alpha=0.05$ is the type I error, $v$ is the degree freedom for error, and $\mathrm{s}^{2}$ is the MSE. The number covered is equal the number of times the simulated parameters satisfied the inequality. The true coverage level is $\frac{1}{1000}$ (Number Covered $) \times 100 \%$.

\section{2.c Reparameterization}

Rewriting a nonlinear model in its expected value parameterization can greatly reduce the model's parameter effects curvature. The expected value parameterization technique advocated by Ratkowsky (1990) has many other advantages, such as: estimators are more normally distributed, less biased, and less correlated; standard errors of estimators are more precise; and the model converges more quickly. Therefore, this method was used to reparameterize the 3-parameter Freundlich model. We decided to replace only $\delta$ and $\beta$ with their expected value equivalent for two reasons: First, $\tau$ has good nonlinear properties; Secondly, it's impossible to replace all three parameters with their expected value equivalent simultaneously for 3parameter Freundlich model.

The following steps are used to get the expected value parameterization for 3parameter Freundlich model. First, we defined $\mu_{1}$ and $\mu_{2}$ as the predicted values of $Y$ at Xmin and Xmax, respectively.

$$
\begin{array}{ll}
\mu_{1}=\delta+\beta X_{1}^{\tau}, & X_{1}=X \min \\
\mu_{2}=\delta+\beta X_{2}^{\tau}, & X_{2}=X \max
\end{array}
$$

Solving these two equations for $\delta$ and $\beta$ leads to

$$
\begin{gathered}
\delta=\left(\mu_{1} X_{2}^{\tau}-\mu_{2} X_{1}^{\tau}\right) /\left(X_{2}^{\tau}-X_{1}^{\tau}\right) \\
\beta=\left(\mu_{2}-\mu_{1}\right) /\left(X_{2}^{\tau}-X_{1}^{\tau}\right)
\end{gathered}
$$

After substituting back into Freundlich model and rearranging, we get the final expected value parameterization model:

$$
Y=\mu_{1}+\frac{\mu_{2}-\mu_{1}}{X_{2}^{\tau}-X_{1}^{\tau}}\left(X^{\tau}-X_{1}^{\tau}\right)+\varepsilon \quad \varepsilon \sim N\left(0, I \sigma^{2}\right)
$$

\subsection{Designs}

The viscometer used to measure shear stress in the study only has 6 rotational speeds, which are 1, 2.5, 5, 10, 20, $50 \mathrm{rpm}$ (Voss, 2002). An interesting question is whether or not the design is optimal. If the design is not optimal, then finding an optimal design for the constraints of the viscometer would be useful.

The shear strain rate, $\mathrm{X}$, is a linear function of the rotational speed. Assuming the rotational speed can be any value between 1 and $50 \mathrm{rpm}$, then $\mathrm{X}$ can be any between 
0.21 and 10.93. In total, we considered four different designs, which are shown in Table 2.

The D-optimal design was applied to nonlinear regression models by Box and Lucas (1959). Their procedure is to: First, pick the initial design using $\mathrm{P}$ points for a Pparameter nonlinear model. Then maximize the determinant of $\mathrm{V}^{0}$, which is the first derivative evaluated at some specified initial parameter estimates. Finally, repeat the design points to get more precise estimates. In our study, the determinant of $\mathrm{V}^{0}$ depends only on $\tau$ and $\tau$ generally takes values between 0.3 and 0.8 . We set $\tau$ equal to 0.55 and got three experimental points, which were $0.21,2.94$, and 10.93 . The final D-optimal design was obtained by repeating the three points once. For the modified D-optimal design, one 10.93 point was changed to 4.37 for two reasons: 1) To estimate intrinsic curvature, at least four different points are needed for 3-parameter nonlinear model; 2) Shear stress, Y, at Shear Strain Rate, X, equal to 10.93 is difficult to observe and is sometimes missing.

\subsection{Assessing Nonlinear Behavior}

Least squares estimation of parameters in nonlinear regression needs two assumptions: planarity and uniformity of coordinates. Bates and Watts (1980) proposed relative measures for intrinsic and parameter-effects curvature to assess how close a modeldata set combination is to satisfying these asymptotic properties. Intrinsic curvature (IN) measures the relative curvature of the expectation surface at the point of convergence. IN increases as the curvature of the expectation surface increases, invalidating the linear estimation procedure. Large IN values indicate unacceptable deviation from the tangent plane assumption. Parameter-effects curvature, PE, measures the lack of uniformity of the parameter lines on the tangent plane. A higher $\mathrm{PE}$ indicates higher degree of departure from the assumption of parallelism. The square root of the average squared curvature can be standardized by multiplying by

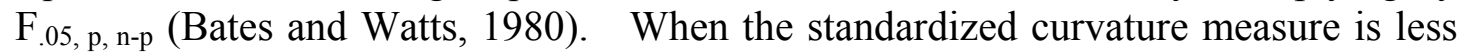
than or equal to 0.4 , the curvatures are considered acceptable.

Ratkowsky (1990) suggests examining the close-to-linear behavior of each parameter. The measures used in this paper are: Box's approximate measure of bias (1972), percent excess variance based on Lowry and Morton' s asymmetry measure (1983), and Hougaard's approximate measure of skewness (1985). Using the under 1\% ruleof-thumb for absolute \%bias suggests all parameters appear to have close-to-linear behavior. Lowry and Morton's asymmetry measure can be expressed as percentage of excess variance. Ratkowsky (quoted in Seber and Wild, 1989, p 188) gives reference values for the measure. Expressed as percentage, values $<1 \%$ indicate behavior is very close to linear. Skewness measures departure from symmetry. Hougaard's approximate skewness works well when $\mathrm{n}>150$. Ratkowsky (1983) gives reference values for skewness. They are: $\mid$ skewness $\mid<0.1$ indicates behavior is very close to linear; between 0.1 and 0.25 , reasonably close to linear; between 0.25 and 1 , apparent skewness; and $>1$, considerable skewness. Programs written in PROC IML (SAS, 1999) were used to estimate intrinsic and parameter effects curvature (IN and PE) and close-to-linear behavior (\%Bias, \%excess variance and skewness). 


\section{RESULTS AND DISCUSSION}

The problems with the conventional method of estimating the parameters of the Herschel-Bulkley model are illustrated in Table 3. The true values of the parameters are those used in the simulation. The apparant standard errors (SE) are a function of the first derivatives evaluated at the true parameters. Traditionally, visual estimation is used to estimate yield stress, $\delta$. We used linear regression which we would expect to be more accurate then the visual estimates. But, even this objective method is highly biased (44.4\%). Furthermore, there is no estimate of the standard error and no possibility for statistical inference. Traditionally, the visual estimate of $\delta$ is then removed from the Herschel-Bulkley model. The consistency coefficient, $\beta$, and flow behavior index, $\tau$, are then estimated from a linearization of the reduced HerschelBulkley. The result shows that $\beta$ and $\tau$ are highly biased $(>>1 \%)$ and the precision is less than ideal. The standard errors of $\beta$ and $\tau$ are greater than the apparant standard errors. Moreover, the MSE is also highly biased $(>>1 \%)$ and the estimated MSE (1.349) is much greater than the true MSE (0.00684).

The Herschel-Bulkley model is a Freundlich model with an additional parameter for the Y-intercept. Using fixed effects nonlinear parameter estimation on the original design provides a good approximation to the rheological properties. All six observations are close to the predicted curve (Figure 3), the MSE (0.00684) is small and the $95 \%$ confidence intervals exclude zero. Yet, the correlations among estimators are as high as 0.99 , which may cause some problems (Table 4) and the nonlinear behaviors are less than ideal. The parameter effects curvature is 8.78 , which is much greater than the critical value of 0.4 (Table 5). Table 8 shows $\delta$ and $\beta$ have high percent bias and excess variance.

On the other hand, when the expected value parameterization of the Freundlich model is used with original design the fit is as before and the nonlinear behavior improves. The PE is 0.47 , which is only slightly greater than 0.4 , and the \%bias, \%excess variance and skewness are satisfactory for all parameters (Table 8). Also, correlations among the estimators for the expected value parameterization are much lower than those for Freundlich model. Some correlations are as low as 0.1, while the highest correlation (0.68) appeared between $\mu_{1}$ and $\tau$ (Table 4).

The SCI adjusted with Bonferroni method and JCR for both models with original design are illustrated in Figures 4 and 5. The cubes are SCI and the ellipsoids are JCR. The big difference between the cube and ellipsoid indicates that it is inappropriate to use SCI instead of JCR for Freundlich model. On the other hand, the difference between SCI and JCR of expected value parameterization is much smaller than that of Freundlich model. The results of the simulation study used to compare coverage levels are given in Table 6. The true coverage levels of 95\% JCR for Freundlich model and its expected value parameterization are $92.2 \%$ and $95.7 \%$, respectively; the true coverage levels of $95 \%$ unadjusted SCI for Freundlich model and its expected value parameterization are $94.4 \%$ and $90.8 \%$, respectively; the true coverage levels of 95\% JCR adjusted with Bonferroni method for Freundlich model and its expected value parameterization are $98.2 \%$ and $97.2 \%$, respectively. Therefore, $95 \%$ joint confidence region for expected value parameterization is more reliable. Also, the true coverage level of $95 \%$ SCI adjusted with Bonferroni method is much closer to that of 95\% JCR for expected value parameterization than for Freundlich model. 
The precision and nonlinear behaviors of the three new designs were compared to those of the original design. The expected precision is given in Table 7. The Doptimal design is optimal since it minimizes the volume of the joint inference region. Therefore, it is expected to have the best precision. The modified D-optimal design, which was introduced to estimate intrinsic curvature, compares favorably with the Doptimal design for the expected value parameterization and is even better than the Doptimal design for the original parameterization of the Freundlich model. Both designs provide an improvement over the original. However, the equally spaced design, a common design for linear models, has slightly less precision than the original design.

The nonlinear behaviors for all designs are given in Table 8. The results for the new designs compared to the original parameterization of the Freundlich model were disappointing. Although there are some improvements in PE, bias and excess variance, all behaviors still exceed the criteria (Tables 5 and 8). The modified Doptimal design has the smallest parameter effects curvature, 6.42, which is still much greater than 0.4. The estimates for the three additional designs are also highly correlated and provide no obvious improvement over the original design (Table 4). However, the additional designs did prove useful for the expected value parameterization, especially the modified D-optimal design, where PE equals 0.22 , which is less than half of that for original design. For all estimators, percent bias, percent excess variance and skewness are very small and most of them are close to 0 . In addition, the correlations among the parameters are remarkably smaller (Table 4). Therefore, the modified D-optimal design with expected value parameterization is recommended. Its precision is comparable to the D-optimal design; its intrinsic curvature can be estimated; and its nonlinear behavior is excellent.

\section{SUMMARY}

Herschel-Bulkley model is an extension of Freundlich model to a three-parameter model: $\delta$ is yield stress; $\beta$ is consistency coefficient; and $\tau$ is flow behavior index. The conventional method which depends on the visual estimation of $\delta$ is problematic. The $\delta$ will have high bias, no standard error and no possibility for statistical inference. Also the estimation of $\beta$ and $\tau$ will be affected. In addition the linearization of the Freundlich model will lead to high bias as well. The nonlinear behaviors of Freundlich model are not ideal; its parameter effects curvature is much greater than 0.4. The high bias and excess variance of $\delta$ and $\beta$ result in the high PE. On the other hand, the Expected Value Parameterization has good nonlinear behavior. Its PE is only slightly greater than 0.4 . Furthermore, the bias, excess variance and skewness for all estimators satisfy the criteria $(<1 \%)$. New designs are not very useful for the original parameterization of the Freundlich model in this experiment. Although there is some improvements in PE, bias and excess variance, all still exceed the criteria. However, new designs are useful for Expected Value Parameterization, especially the modified D-optimal design, where PE is less than half of that for original design. The percent bias, percent excess variance and skewness of all estimators are very small, most of them are close to 0 . Therefore, we recommend using the Expected Value Parameterization with modified D-optimal design to obtain excellent nonlinear behavior. For future research on rheological properties, our study provides an efficient design and a nonlinear procedure to get accurate estimates of rheological parameters of yogurt, thus, enabling researchers to get better comparisons of the effects of chemical additives and storage time on rheological behavior of yogurt. 


\section{REFERENCES}

Bates, D. M. and D. L. Watts. 1980. "Relative curvature measures of nonlinearity." J. R. Statist. Soc. Ser. B. 42:1-25.

Box, G. E. P., and H. L. Lucas. 1959. "Design of experiments in non-linear situations." Biometrika. 46: 77-90.

Box, M. J. 1972. “ Bias in nonlinear estimation.” J. R. Statist. Soc. Ser. B. 33:171201.

Hougaard, P. 1985. "The appropriateness of the asymptotic distribution in a nonlinear regression model.” J. R. Statist. Soc. Ser. B. 47:103-114.

Lowry, R. and R. Morton. 1983. "An asymmetry measure for estimators in nonlinear regression models." Proc. $44^{\text {th }}$ Session Int. Statist. Inst., Madrid, Contributed Papers 1:351-354.

Ratkowsky, D. A. 1983. Nonlinear Regression Modeling: A Unified Practical Approach. Marcel Dekker: New York and Basel.

Ratkowsky, D. 1990. Handbook of nonlinear regression models. Marcel Dekker, New York and Basel.

SAS. 1999. SAS/STAT User' s Guide. Version 8. SAS Institute Inc., Cary, NC.

Seber, G. and C. Wild, 1989. Nonlinear Regression. John Wiley \& Sons, New York.

Steffe, J. F. 1996. Rheological Methods in Food Process Engineering. Freeman Press, East Lansing.

Voss, H. K. 2002. The Functional Properties of Fructooligosaccharides in Nonfat Yogurt. Thesis (M.S.). University of Nebraska--Lincoln. 
Figure 1 Examples of Shear Stress,Y, vs Shear Strain Rate, $X$, for a Range of Flow Behavior

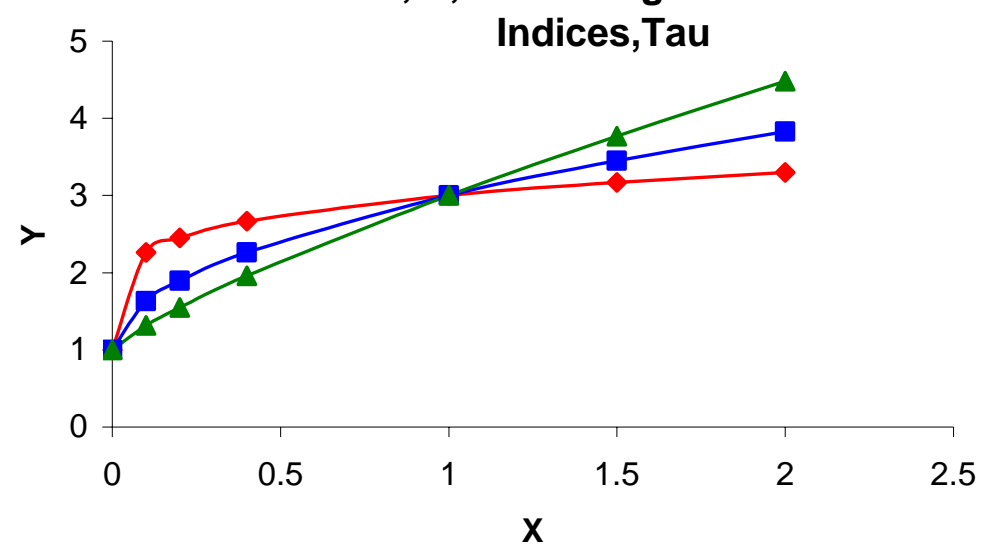

Figure 2 Examples of Shear Stress, Y, vs Shear Strain Rate, $X$, for a Range of Consistency Coefficients, Beta
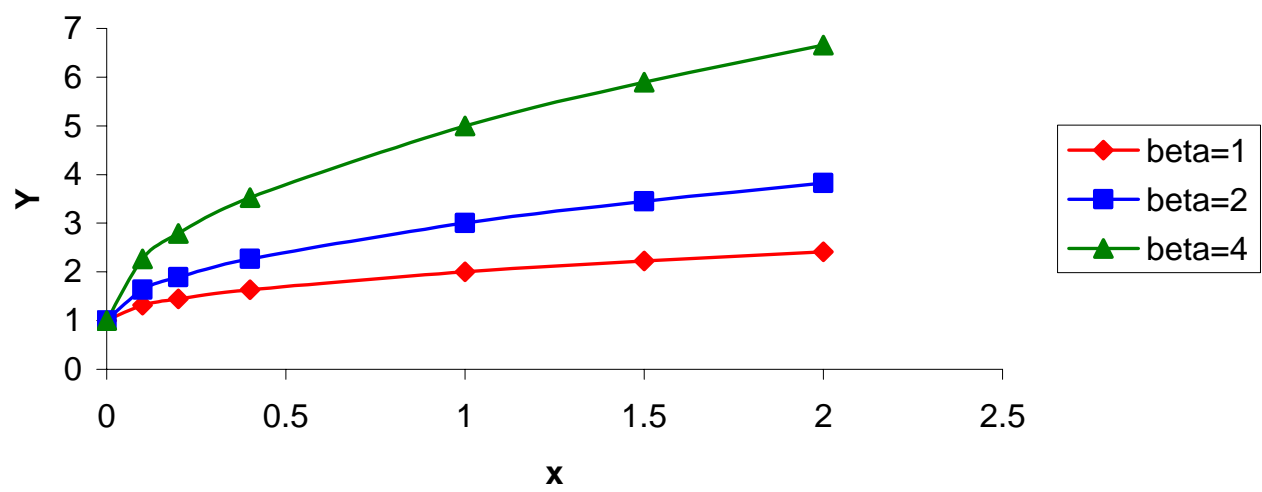

Figure 3 Freundlich Model for Original Design

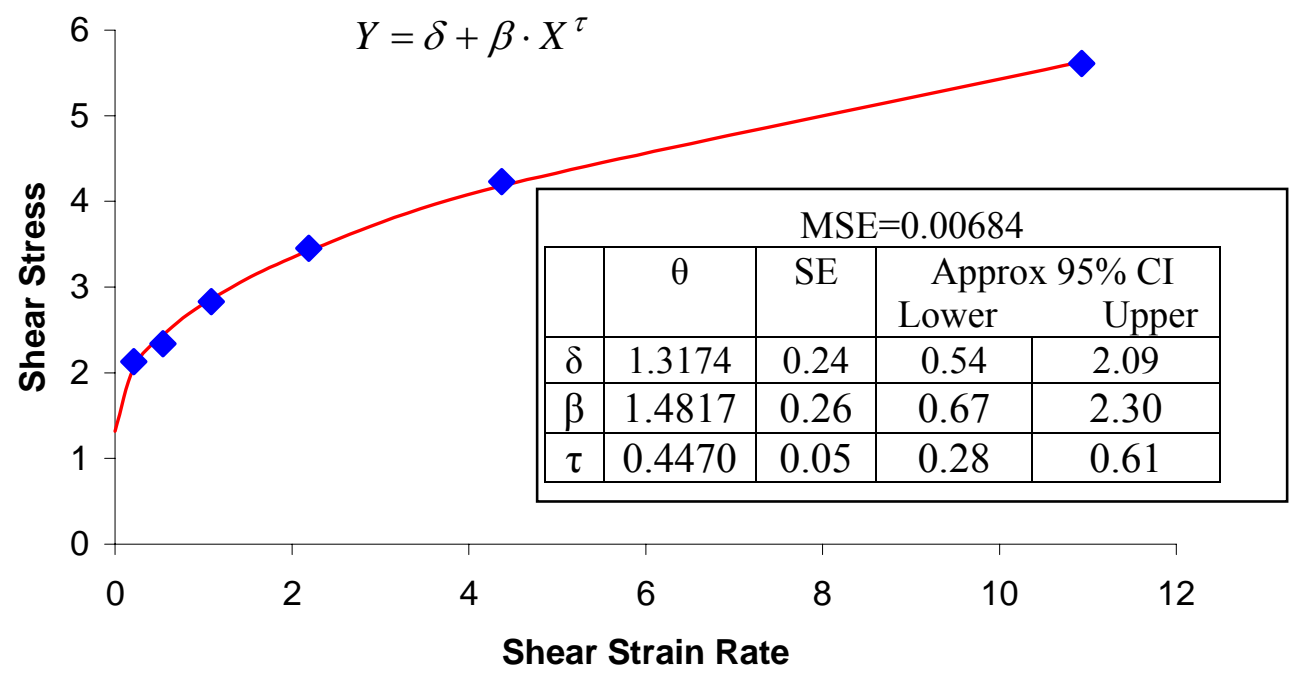


Figure 4 95\% Simultaneous Confidence Interval and Joint Confidence Region for Freundlich Model

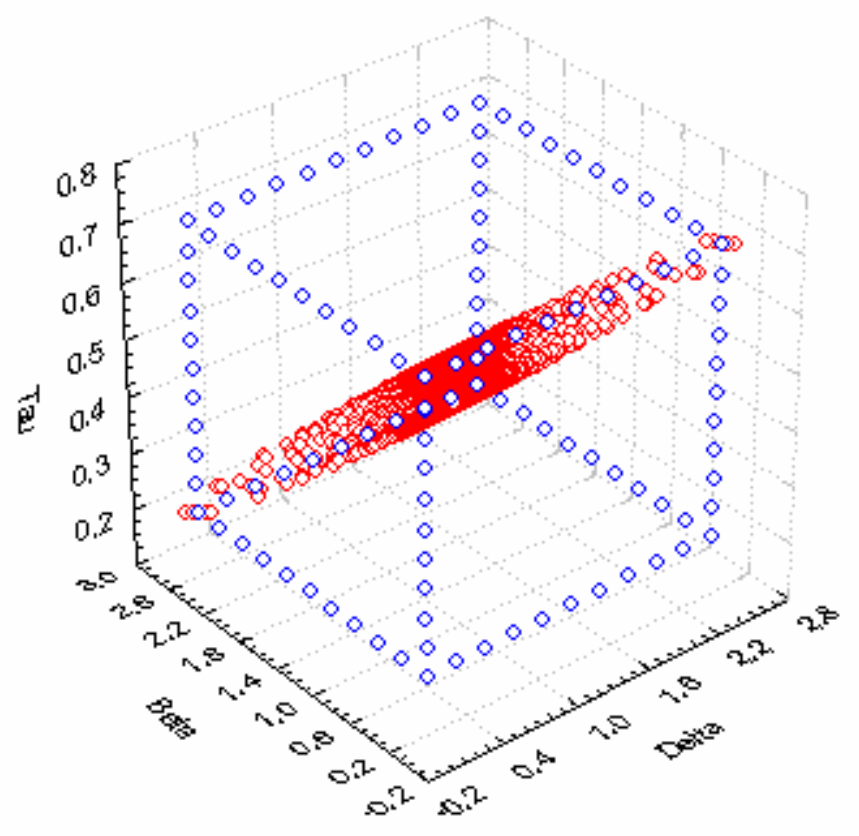

Figure 5 95\% Simultaneous Confidence Interval and Joint Confidence Region for Expected Value Parameterization

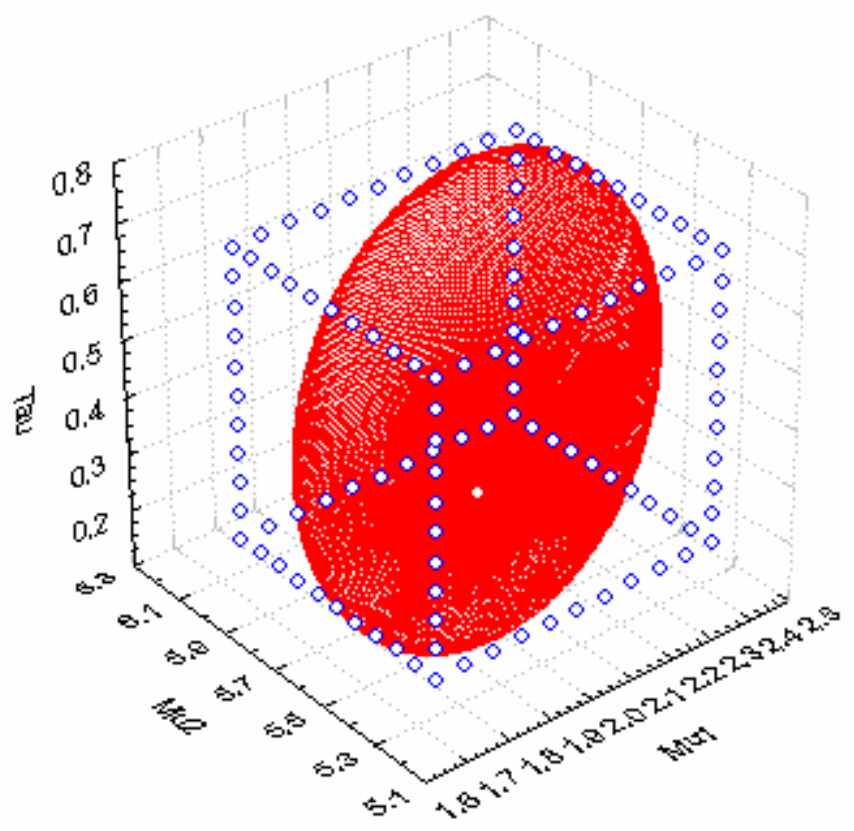


Table 1 Data Used to Estimate Rheological Properties of Yogurt

\begin{tabular}{|c|c|}
\hline Shear strain rate $(1 / \mathrm{sec})$ & Shear stress $(\mathrm{Pa})$ \\
\hline 0.21 & 2.13 \\
\hline 0.54 & 2.34 \\
\hline 1.09 & 2.83 \\
\hline 2.19 & 3.45 \\
\hline 4.37 & 4.23 \\
\hline 10.93 & 5.61 \\
\hline
\end{tabular}

Table 2 Experimental Designs for Shear Strain Rate

\begin{tabular}{|c|c|}
\hline Design & $\mathrm{X}$ \\
\hline Original & $0.21,0.54,1.09,2.19,4.37,10.93$ \\
\hline Equally Spaced & $0.21,2.354,4.498,6.642,8.786,10.93$ \\
\hline D-optimal & $0.21,0.21,2.94,2.94,10.93,10.93$ \\
\hline Modified D-optimal & $0.21,0.21,2.94,2.94,4.37,10.93$ \\
\hline
\end{tabular}

Table 3 Estimates and Bias for Conventional Procedure

\begin{tabular}{|c|c|c|c|c|c|}
\hline Parameter & True Value & $\begin{array}{l}\text { Apparant } \\
\text { SE* }^{*}\end{array}$ & Estimate & Estimated SE & $\begin{array}{c}\text { Relative Bias } \\
(\%)\end{array}$ \\
\hline$\delta$ & 1.3174 & 0.24 & 1.902 & ---- & 44.4 \\
\hline$\beta$ & 1.4817 & 0.26 & 0.711 & 1.14 & -52.0 \\
\hline$\tau$ & 0.4470 & 0.05 & 0.794 & 0.09 & 77.7 \\
\hline$\sigma^{2}$ & 0.00684 & 0.0827 & 1.349 & 1.161 & 196.2 \\
\hline
\end{tabular}

* Based on the true values of the parameters.

Table 4 Correlations among Parameters

\begin{tabular}{|c|c|c|c|c|c|c|c|c|}
\hline Parameterization & \multicolumn{4}{|c|}{ Original Freundlich } & \multicolumn{4}{|c|}{ Expected Value } \\
\hline Design & & $\delta$ & $\beta$ & $\tau$ & & $\mu_{1}$ & $\mu_{2}$ & $\tau$ \\
\hline \multirow{3}{*}{ Original } & $\delta$ & 1 & -0.99 & 0.96 & $\mu_{1}$ & 1 & 0.10 & 0.68 \\
\hline & $\beta$ & & 1 & -0.99 & $\mu_{2}$ & & 1 & 0.48 \\
\hline & $\tau$ & & & 1 & $\tau$ & & & 1 \\
\hline \multirow{3}{*}{ Equally Spaced } & $\delta$ & 1 & -0.97 & 0.93 & $\mu_{1}$ & 1 & 0.07 & 0.52 \\
\hline & $\beta$ & & 1 & -0.99 & $\mu_{2}$ & & 1 & 0.60 \\
\hline & $\tau$ & & & 1 & $\tau$ & & & 1 \\
\hline \multirow{3}{*}{ D-optimal } & $\delta$ & 1 & -0.97 & 0.94 & $\mu_{1}$ & 1 & 0 & 0.44 \\
\hline & $\beta$ & & 1 & -0.99 & $\mu_{2}$ & & 1 & 0.38 \\
\hline & $\tau$ & & & 1 & $\tau$ & & & 1 \\
\hline \multirow{3}{*}{ Modified D-optimal } & $\delta$ & 1 & -0.98 & 0.93 & $\mu_{1}$ & 1 & 0.01 & 0.42 \\
\hline & $\beta$ & & 1 & -0.98 & $\mu_{2}$ & & 1 & 0.60 \\
\hline & $\tau$ & & & 1 & $\tau$ & & & 1 \\
\hline
\end{tabular}


Table 5 Measures of Curvature

\begin{tabular}{|c|c|c|c|c|}
\hline \multirow{2}{*}{ Design } & \multicolumn{4}{|c|}{ Parameterization } \\
\cline { 2 - 5 } & \multicolumn{2}{|c|}{ Freundlich } & Expected Value \\
\cline { 2 - 5 } & IN & PE & IN & PE \\
\hline Original & 0.18 & 8.78 & 0.18 & 0.47 \\
\hline Equally Spaced & 0.12 & 12.50 & 0.12 & 0.28 \\
\hline D-optimal & N/A & 8.0 & N/A & 0.22 \\
\hline Modified D-optimal & 0.05 & 6.42 & 0.05 & 0.22 \\
\hline
\end{tabular}

Table 6 True Coverage Level

\begin{tabular}{|c|c|c|}
\hline \multirow{2}{*}{ Nominal Coverage Level } & \multicolumn{2}{|c|}{ \%True Coverage Level } \\
\cline { 2 - 3 } & Freundlich & Expected Value \\
\hline Unadjusted 95\% SCI & 94.4 & 90.8 \\
\hline 95\% SCI Adjusted with Bonferroni Method & 98.2 & 97.2 \\
\hline 95\% JCR & 92.2 & 95.7 \\
\hline
\end{tabular}

Table 7 Expected Precision: Coefficients for Covariance among Parameters

\begin{tabular}{|c|c|c|c|c|c|c|c|c|}
\hline Parameterization & \multicolumn{4}{|c|}{ Original Freundlich } & \multicolumn{4}{|c|}{ Expected Value } \\
\hline Design & & $\delta$ & $\beta$ & $\tau$ & & $\mu_{1}$ & $\mu_{2}$ & $\tau$ \\
\hline \multirow{3}{*}{ Original } & $\delta$ & 8.69 & -9.00 & 1.79 & $\mu_{1}$ & 0.73 & 0.09 & 0.37 \\
\hline & $\beta$ & & 9.57 & -1.93 & $\mu_{2}$ & & 0.94 & 0.29 \\
\hline & $\tau$ & & & 0.40 & $\tau$ & & & 0.40 \\
\hline \multirow{3}{*}{ Equally Spaced } & $\delta$ & 8.92 & -9.08 & 1.80 & $\mu_{1}$ & 0.98 & 0.05 & 0.33 \\
\hline & $\beta$ & & 9.73 & -1.98 & $\mu_{2}$ & & 0.62 & 0.31 \\
\hline & $\tau$ & & & 0.42 & $\tau$ & & & 0.42 \\
\hline \multirow{3}{*}{ D-optimal } & $\delta$ & 5.64 & -6.11 & 1.22 & $\mu_{1}$ & 0.5 & 0 & 0.17 \\
\hline & $\beta$ & & 6.97 & -1.42 & $\mu_{2}$ & & 0.5 & 0.15 \\
\hline & $\tau$ & & & 0.30 & $\tau$ & & & 0.30 \\
\hline \multirow{3}{*}{ Modified D-optimal } & $\delta$ & 5.34 & -5.67 & 1.17 & $\mu_{1}$ & 0.5 & 0 & 0.16 \\
\hline & $\beta$ & & 6.33 & -1.34 & $\mu_{2}$ & & 0.98 & 0.32 \\
\hline & $\tau$ & & & 0.30 & $\tau$ & & & 0.30 \\
\hline
\end{tabular}


Table 8 Measures of Nonlinear Behavior: \%Bias, \%Excess Variance and Skewness

\begin{tabular}{|c|c|c|c|c|c|c|c|c|}
\hline Model & \multicolumn{4}{|c|}{ Freundlich } & \multicolumn{4}{c|}{ Expected Value } \\
\hline Design & Parm & Bias & ExVar & Skew & Parm & Bias & ExVar & Skew \\
\hline \multirow{3}{*}{ Original } & $\delta$ & -1.94 & 2.30 & -0.58 & $\mu 1$ & -0.03 & 0.07 & -0.03 \\
\cline { 2 - 10 } & $\beta$ & 1.77 & 2.20 & 0.60 & $\mu 2$ & 0.01 & 0.03 & 0.01 \\
\cline { 2 - 9 } & $\tau$ & 0.17 & 0.24 & 0.09 & $\tau$ & 0.17 & 0.24 & 0.09 \\
\hline \multirow{3}{*}{ Equally Spaced } & $\delta$ & -2.03 & 2.39 & -0.56 & $\mu 1$ & -0.01 & 0.01 & 0 \\
\cline { 2 - 9 } & $\beta$ & 1.90 & 2.43 & 0.64 & $\mu 2$ & 0.01 & 0.03 & 0.02 \\
\cline { 2 - 9 } & $\tau$ & 0.10 & 0.15 & 0.05 & $\tau$ & 0.10 & 0.15 & 0.05 \\
\hline \multirow{3}{*}{ D-optimal } & $\delta$ & -1.42 & 1.81 & -0.51 & $\mu 1$ & 0 & 0 & 0 \\
\cline { 2 - 9 } & $\beta$ & 1.33 & 1.62 & 0.53 & $\mu 2$ & 0 & 0 & 0 \\
\cline { 2 - 9 } optimal & $\tau$ & 0.09 & 0.07 & 0.05 & $\tau$ & 0 & 0.07 & 0.05 \\
\hline & $\delta$ & -1.44 & 1.98 & -0.52 & $\mu 1$ & 0 & 0 & 0 \\
\cline { 2 - 9 } & $\beta$ & 1.35 & 1.86 & 0.56 & $\mu 2$ & 0 & 0 & 0 \\
\hline \multirow{3}{*}{\begin{tabular}{c} 
Modified D- \\
\cline { 2 - 8 }
\end{tabular}} & $\tau$ & -0.01 & 0.08 & -0.003 & $\tau$ & -0.01 & 0.08 & 0 \\
\hline
\end{tabular}

